\title{
P02-238
}

\section{COMPARISON BETWEEN PREVALENCE AND PATTERNS OF CIGARETTE SMOKING AMONGST SIXTH FORM STUDENTS IN THE UNITED KINGDOM AND PAKISTAN}

M. Husain ${ }^{1}$, S. Khan ${ }^{2}$, M. Husain ${ }^{3}$, M. Saeed ${ }^{4}$, N. Chaudhry ${ }^{4}$

${ }^{1}$ St. George's, University of London, London, ${ }^{2}$ University of Manchester, Manchester, ${ }^{3}$ Farnham Road Hospital, London, ${ }^{4}$ University of Manchester, Manchester, UK

Background: Tobacco smoking is one of the major preventable causes of premature death and disease in the world. Adolescents are amongst the most vulnerable group of individuals in society and are highly susceptible to cigarette smoking. Sixth Formers being the most senior members of the school act as role models to younger pupils and could influence smoking behaviour.

Objective: In a cross sectional and comparative study we aimed to determine the differences in smoking prevalence and patterns between Sixth Form students in Pakistan (a developing country) and the United Kingdom (a developed country).

Method: A self-administered questionnaire, adapted from the WHO questionnaire, about tobacco smoking for health professionals was distributed amongst the students. The responses were anonymised to encourage truthful answers; there was no pressure to respond.

Of the 235 participants in the UK, $38(16 \%)$ were regular smokers. Of the 297 participants in Pakistan, $20(7 \%)$ of participants were regular smokers. In both countries there was a significant difference between the genders, with males being more likely to smoke. In Pakistan it was identified that the main reason for smoking was due to peer pressure, where as in the UK it was for recreational and social purposes.

Conclusions: The prevalence of smoking was higher in UK compared to Pakistan. There was a significant difference in the smoking behaviour patterns in the two countries. 